\title{
L'influence De La Contrebande Sur Le Developpement De La Ville Frontaliere De Niable Au Centre-Est De La Cote d'Ivoire
}

\author{
Atta Koffi \\ Maître de Recherches à l'Institut de Géographie Tropicale, \\ Université Félix Houphouët Boigny de Cocody, Abidjan, Côte d’Ivoire \\ Gogbe Téré \\ Maitre de Conférences à l’Institut de Géographie Tropicale, \\ Université Félix Houphouët Boigny de Cocody-Abidjan, Côte d’Ivoire \\ Kouassi Nguessan Gilbert \\ Assistant à l'institut de Géographie Tropicale, \\ Université Felix Houphouet Boigny de Cocody-Abidjan, Côte d’Ivoire

\section{Kouadio Datté Anderson} \\ Doctorant à l'Institut de Géographie Tropicale, \\ Université Félix Houphouët Boigny de Cocody-Abidjan, Côte d’Ivoire
}

doi: 10.19044/esj.2016.v12n5p313 URL:http://dx.doi.org/10.19044/esj.2016.v12n5p313

\begin{abstract}
In the border town of Niablé, smuggling is omnipresent. The operation of this activity is based on the one hand, on social cultural relationship between neigh boring populations, and on the other hand on monetary disparities between Ghana and Côte d'Ivoire. Without denying the truth fullness of such an activity, smugglers, organized in a highly hierarchical system operate according to a strict behavioral code where one's given word and mutual confidence are the sole modus operandi. It permits them to bring goods of diverse natures (oil products, food, pharmaceuticals, second-hand clothes drinks, etc.) in Niablé. Thus, this activity seems to be a real opportunity for these smugglers, longing for the improvement of their welfare. Besides, the smuggling allows its actors to contribute to the town's blossoming. Such a perception of smuggling gives an idea of the animation going on in this border town. In a word, though we want it or not smuggling participates in the socio-spatial change of the town even if sometimes, it stands as an obstacle to its development.

Through this contribution, we are willing to show the operation of smuggling and its influence on the town of Niablé.
\end{abstract}


Keywords: Smugglers, border, network, Niablé, Ghana, Ivory Coast

\section{Resume}

Dans la ville frontalière de Niablé, la contrebande est omniprésente. Le fonctionnement de cette activité repose d'une part sur les relations socioculturelles entre les population frontalières et d'autre part sur la disparité monétaire entre la Ghana et la Côte d’Ivoire. Sans nier la véracité d’une telle activité, les contrebandiers, fortement hiérarchisés, opèrent selon un code de conduite strict où la parole donnée et la confiance mutuelle sont le mode operatoire. Ce qui leur permet de faire rentrer des marchandises de diverses natures (produits pétroliers, alimentaires, pharmaceutiques, de la friperie, de la boisson etc.) à Niablé. Ainsi, cette activité se présente comme une réelle opportunité pour les contrebandiers à la course de l'amélioration de leur bien-être. Outre, la contrebande permet à ses acteurs d'y contribuer à l'épanouissement urbain. Cette perception de la contrebande donne une idée de l'animation frontalière de la ville. En un mot, qu'on le veuille ou non, la contrebande participe à la mutation socio-spatiale de la ville même si parfois elle se pose comme un frein à son développement.

A travers cette contribution, nous montrons l'organisation de la contrebande et son influence sur la ville de Niablé.

Mots clé : Contrebandier, douanier, frontière, Niablé, Ghana, Côte d’Ivoire

\section{INTRODUCTION}

La Cote d'Ivoire et le Ghana partagent une frontière terrestre longitudinale de $670 \mathrm{~km}$ (Nassa, 2005). Dans cet espace brouille un bourdonnement d'activités contrebandières perturbant le plus souvent le développement des localités frontalières. C'est le cas de la ville frontalière de Niablé, où l'agitation commerciale frontière engendre une mobilité de plusieurs individus ayant relativement les mêmes objectifs. En effet, Niablé est une localité située à l'Est-forestier de la Côte d'Ivoire et distante de $3 \mathrm{~km}$ de la frontière ghanéenne (cf. figure 1). Cette ville frontalière, support d'une effervescence commerciale se positionne aussi comme un point un passage obligé pour les commerçants frontaliers. Pourtant, elle n’est pas seulement le siège d'échanges de proximité officiel, mais elle sert également d'appui et de relais pour des flux illégaux: on parle de la contrebande. D’ailleurs, la vitalité de cette activité illicite dans la localité prend l'allure d'une foire où plusieurs individus d'origines et de fonctions diverses se rencontrent pour opérer des échanges de toutes natures. Base-arrière des exploitants de la frontière, Niablé représente le lieu où les contrebandiers supervisent et dé cident de l' organisation et de la ventilation de leur flux en direction des 
marchés nationaux. La ville apparait ainsi comme le lieu du possible, un espace-exutoire où les contrebandiers viennent parier et risquer leur chance. Leur activité est considérée comme une «institution locale» pour la population frontalière qui y fait recours quotidiennement pour sa survie. La contrebande représente une véritable soupape de sécurité capable de réduire le chômage et offrir des sources de revenus à ses acteurs. Ces derniers soucieux de leur bien-être invertissent le plus souvent dans l'immobilier. Ce qui modifie la trame spatiale de la ville même si les effets pervers d'une telle activité sont aussi visibles dans l'organisation de la ville.

Forte de tout cela, la contrebande se présente comme une activité mutante qui semble avoir un pouvoir d'organisation sociale et spatiale dans la localité qui l'abrite. Alors; l'organisation d'une telle activité à Niablé n'influence t-elle pas le développement de la ville?

Telle est la principale préoccupation que nous allons répondre à travers cette contribution.

Figure1 : Localisation de la ville de Niablé dans sa région administrative en Côte d'Ivoire.

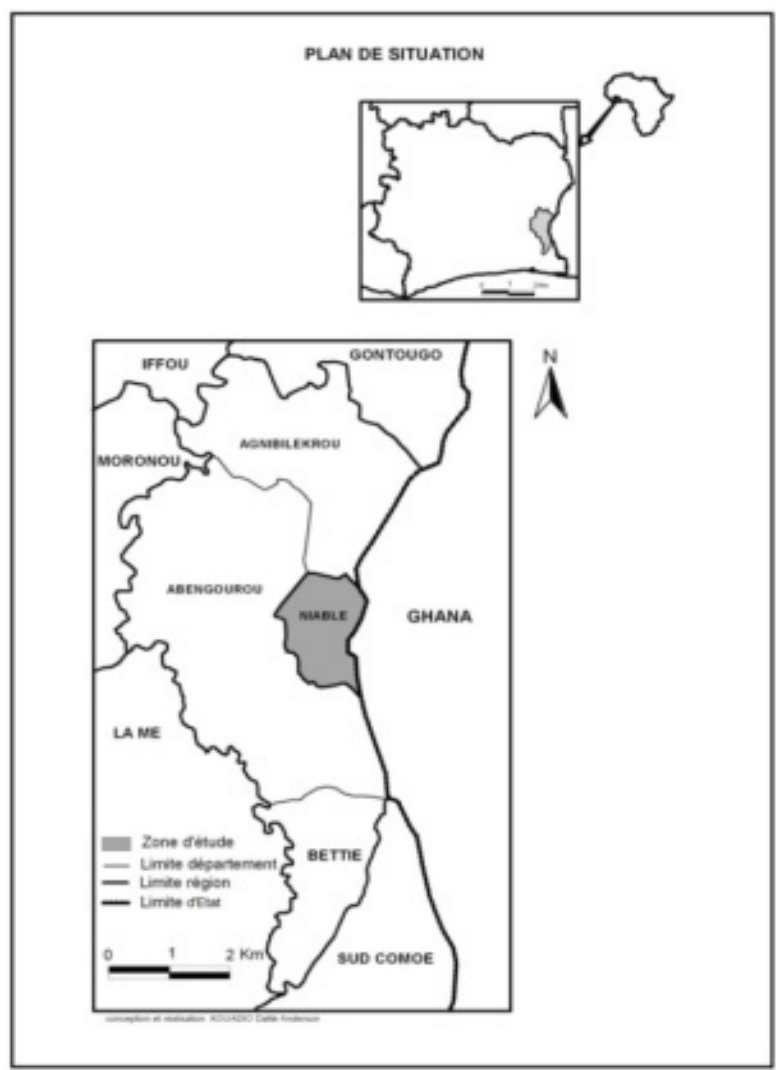




\section{MATERIEL ET METHODE}

Pour mener à bien ce travail, nous avons utilisé la méthode hypothético-déductive afin de décrypter la contrebande ; son organisation et son influence socio-spatiale sur la ville de Niablé. Nous nous sommes appuyés ainsi sur des données primaires et secondaires. Après plusieurs consultations documentaires, nous avons opté pour une enquête de terrain. Ici, nous avons procédé par un sondage non probabiliste c'est-à-dire un choix raisonné des personnes à enquêter. Eu égard la complexité d'une telle activité illicite, nous avons été contraints d'intervenir dans le choix des personnes à interroger. Nous étions surtout dépendant du bon vouloir des enquêtés à nous répondre. C'est pourquoi, nous avons pu interroger que 4 agents de contrôle et 36 contrebandiers. D’ailleurs, les informations dans ce secteur caché étant très réservées, pour percer ce mystère une observation participative a été faite en intégrant différents groupes de contrebandiers. En fait, deux ou trois jours dans la semaine durant notre séjour, nous avons parcouru les pistes clandestines avec les contrebandiers. Dès fois, nous nous sommes rendus avec eux au Ghana par la voie normale, puis nous en sommes revenus souvent par des chemins détournés. Là, nous avons eu des informations sur leurs différentes stratégies à savoir le mode, le lieu d’acquisition, la nature, la qualité et le volume des marchandises.

Un autre volet de cette enquête s’est déroulé auprès des agents de douane avec qui nous avons discuté à bâtons rompus. A la fin, ces agents nous ont autorisés à consulter leurs registres afin d’apprécier la typologie des produits saisis par leur service.

La conjugaison de ces différentes techniques d'approches a pour but de confronter notre étude aux résultats d'études antérieures sur les questions de la contrebande. C'est donc au travers de la combinaison et de l'articulation de toutes ces composantes méthodologiques que prend forme notre méthode globale. Pour cela, nous estimons que trois niveaux sont à creuser pour mesurer les effets de la contrebande sur la ville de Niablé.

\section{RESULTATS ET DISCUSSION}

\section{Les facteurs de la contrebande à Niablé Les facteurs socio-culturels}

En Afrique, l’idée selon laquelle la contrebande est le résultat de la solidarité ethnique des peuples partageant la même langue, civilisation est devenue une sorte de label que l'on accepte sans discussion, selon les travaux de Bennafla (2002). A l'appui de cette vision, il suffit de regarder l'histoire pour comprendre que la solidarité ethnique interétatique dans le contournement des frontières est considérée comme l'un des fondements de la contrebande.

Les populations qui habitent une frontière sont souvent amenées à 
vivre en étroite symbiose de par leurs cultures, leurs langues, leurs valeurs sociales et religieuses qui regroupent des points identiques. En fait, les tracés des frontières ont séparé deux frères, deux sœurs. Elles ont tenté de couper en deux une population africaine très soudée, ayant en commun la même civilisation et les mêmes traditions (Igué, 1997). Dans ces circonstances, à Niablé, la frontière ne peut jamais être un mur entre les deux peuples, quelque soit les micro-nationalismes qui pourraient se développer en Côte d'Ivoire et au Ghana. Les liens socioculturels prolongent les canaux d'échanges frontaliers et apportent des éléments de confiance à des opérations établies au noir. D'ailleurs, la population frontalière des deux côtés exerce des activités commerciales en traversant la frontière souvent sans aucun contrôle. Cette population a le sentiment d'être membre d'une même nation. Alors, la densité des liens socioculturels constitue un élément déterminant dans la performance des échanges surtout ceux relatifs à la contrebande. Outre, cette solidarité culturelle galvanise les contrebandiers qui s'appuient régulièrement sur de nombreuses plantations frontalières ou de créent plusieurs pistes ou chemins clandestins. Ces pistes deviennent par conséquent, un large couloir de passage transversal pour la pratique de cette activité illicite. Ainsi, à chaque village frontalier, il existe plusieurs pistes utilisées par les contrebandiers pour contourner les postes de contrôles ou les corridors de sécurité comme nous le montrons à la figure 2. Toutefois, nous n'avons pas la prétention de cartographier toutes les pistes, nous nous sommes contentés d'en faire les plus fréquentées.

Figure 2: quelques-unes des pistes clandestines traversant la zone frontalière de Niablé

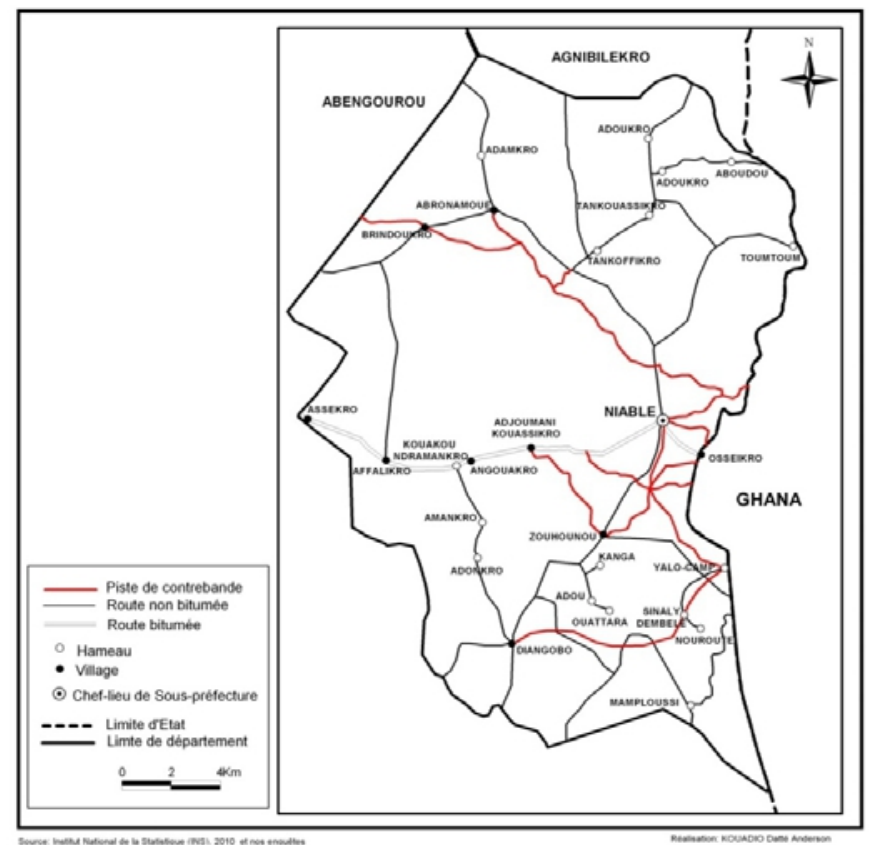




\section{Les facteurs économiques}

A ce niveau, les contrebandiers sont stimulés par trois principales motivations. La première cause, et sans doute l'une des plus anciennes, est la prohibition de certains produits courants. Son effet sur l'essor d'un marché noir est systématiquement observé. Pourquoi ? Car si l'interdiction empêche bel et bien les échanges des produits prohibés sur le marché légal, ceux-ci continuent généralement d'être désirés, recherchés et demandés par les consommateurs. L'absence d'offre légale laisse toute latitude à l'offre de contrebande de s'y substituer. Le marché noir prend alors son essor et la consommation du produit visé ne disparaît pas. A Niablé, les exemples sont nombreux: les produits pharmaceutiques, la drogue; les armes à feu, le carburant, etc.

La deuxième raison évoquée concerne les taxes douanières. En fait, le principe est que l'importation et l'exportation de tous les produits sont libres en Côte d'Ivoire. Cependant, les droits et taxes d'entrée grèvent lourdement le prix de revient des articles importés, selon les contrebandiers. Le prix de revient du produit décourage, le consommateur qui s'oriente vers des articles fabriqués, pour la plupart, en Asie (la chine) et dont l'importation se fait souvent en contrebande. Là où il estime que l'écart entre les prix des produits est à son avantage.

Enfin, la troisième raison est la parité monétaire. En effet, dans notre espace, il coexiste deux zones monnaies: le franc CFA en Côte d'Ivoire et le Cedi au Ghana. Ces deux monnaies n'ont pas le même coût ni la même valeur d'échange. La parité variante de la monnaie ghanéenne sur les marchés locaux et de leur non convertibilité par rapport au franc CFA démontre clairement une dissemblance fiduciaire entre les deux Etats voisins. Ainsi pendant notre séjour le Cedi a subi différentes fluctuations par rapport au CFA comme l'indique le tableau ci-dessous.

Tableau 1 : Parité du franc CFA par rapport au Cedi ghanéen

\begin{tabular}{|l|l|l|}
\hline Période & Franc CFA & Cedi \\
\hline 25 juillet & 1000 & 16000 \\
\hline 5 Août & 1000 & 16500 \\
\hline 16 Août & 1000 & 13000 \\
\hline 21 Août & 1000 & 14500 \\
\hline
\end{tabular}

Source : Notre enquête, Août 2012

Cette disparité loin d’être un obstacle est un parfait stimulant des échanges interétatique et judicieusement exploitée par les contrebandiers.

En somme, force est de souligner que à Niablé l'état poreuse de la frontière concourt à l'éclosion de l'activité contrebandière. Cette porosité doublée par les facteurs socioculturels et économiques alimente fortement la contrebande et lui donne sa raison dans notre espace d'étude. D’ailleurs, ces signaux, nous amène à nous examiner l'organisation de cette activité 
informelle.

\section{Organisation de la contrebande dans l'espace frontalier de Niablé Qui sont les contrebandiers?}

Les contrebandiers sont des individus connectés par des liens d'informations, dont les actions sont associées à un rendement croissant. A Niablé, la contrebande implique les transporteurs, les artisans, les paysans, les jeunes déscolarisés, les enseignants, les hommes en uniformes, quelques fonctionnaires de la ville, retraités ou en activité. Mais elle se montre comme une activité dont l'âge est un atout pour les acteurs. Sur 36 contrebandiers interrogés lors de notre enquête, nous avons 26 individus dont l'âge est compris entre 15 et 30 ans contre 11 qui ont plus de 30 ans. Pour plus de visibilité, nous avons représenté les tranches d’âge des contrebandiers dans un diagramme circulaire (voir figure 2).

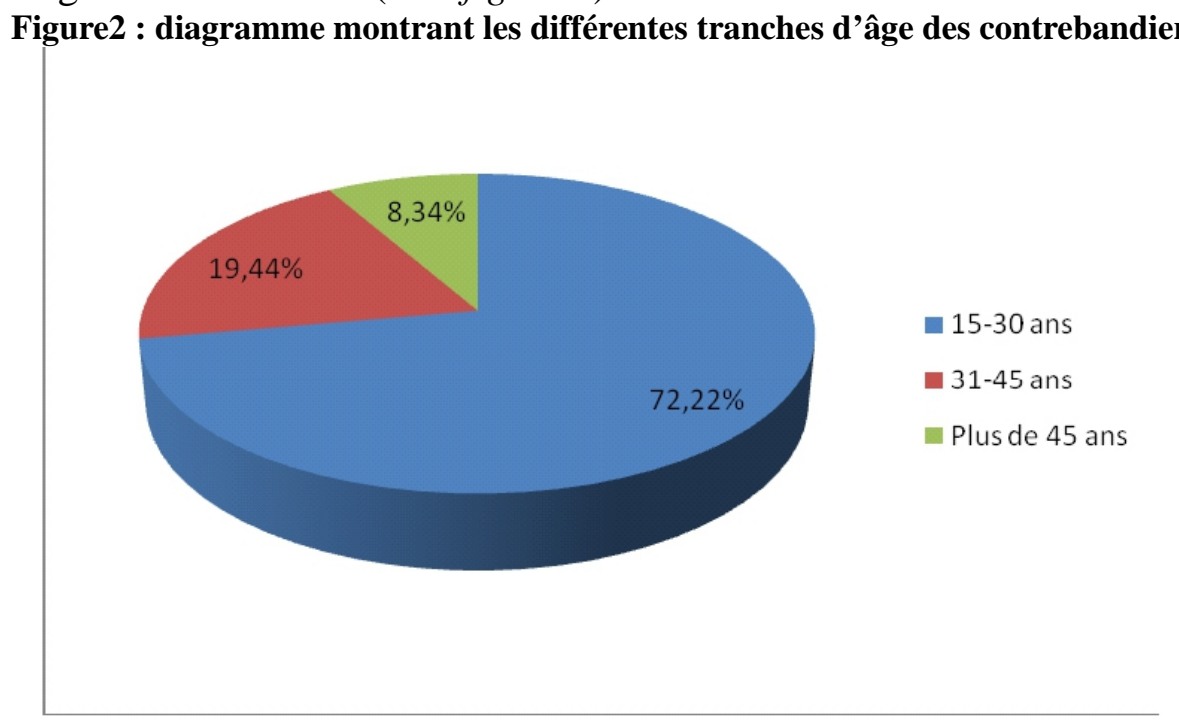

Source : Notre enquête, Aout 2012

A travers le graphique, il n'est pas faut de dire qu'avec un ensemble de 91,66\%, on remarque clairement que les individus de moins de 45 ans occupent une part très importante dans la pratique de la contrebande. Cette catégorie représente les jeunes. En fait, elle demande de la force physique et du courage c'est-à-dire être apte à surmonter les conditions délicates caractérisant cette activité. La contrebande est une activité difficile et dangereuse car le contrebandier, doit avoir un potentiel physique important pour supporter la charge et pouvoir échapper aux agents de contrôle au moment opportun. Donc elle ne peut pas être exercée par n'importe quel âge. 


\section{La typologie des contrebandiers}

\section{Les passeurs ou " trotteurs"}

Ce sont les jeunes natifs de Niablé pour la plupart, dont l'âge varie entre 15 et 35 ans qui s'adonnent à ce type d'activité. Si certains de ces jeunes sont de jeunes planteurs attendant de meilleures rentrées financières pour se consacrer entièrement à l'agriculture, d'autres ont cette activité comme seule source de revenus (Stary, 1994). En réalité, ces jeunes, communément appelés les "trotteurs", ont un réseau de clients fidèles qu’ils retrouvent à la gare routière de Niablé. En effet, une fois que le client et l'équipe se mettent d'accord sur le prix qui varie très souvent entre 5000 à 50.000 FCFA. Ce prix est fixé non seulement en fonction de la tête du client mais aussi de la quantité et de la qualité de la marchandise. Pour l'acheminement de la marchandise au lieu indiqué, les passeurs n’hésitent pas à passer par des pistes clandestines dont ils sont les seuls à connaitre leur existence. Très organisés, opérant en petits groupes de 3 à 6 personnes, ces "trotteurs" se divisent en deux groupes. Le premier groupe joue le rôle de sentinelles en observant les mouvements des agents de contrôle et le deuxième groupe porte les marchandises. Cependant, certains commerçants préfèrent les ignorer en prenant le risque de passer au poste des douanes avec des marchandises indélicates sans déclaration. Pour cela, ils usent de techniques de plus en plus intelligentes pour passer frauduleusement leurs marchandises: c'est la technique du camouflage. Selon un douanier anonyme, cette forme sournoise de fraude est difficile à cerner et à évaluer. Mais avec la complicité de certains commerçants et douaniers, nous avons pu comprendre un temps soit peu ce processus d’illégalité qui se met en œuvre en réalité pendant le retour des commerçants en terre ivoirienne. Evidemment, nous ne saurions déterminer de façon exhaustive la liste des astuces. Celles-ci ont été révélées par certains trafiquants lors des différentes saisies opérées par la douane. Toutes les techniques sont bonnes, pourvu que les agents de contrôle échouent dans leurs tâches régaliennes. Concernant le port des produits prohibés, c'est une technique qui est plus pratiquée par les femmes. Ici la trafiquante dissimule les produits prohibés sous ses habits. A ce niveau, le port d'un collant est indispensable. La trafiquante devient par la même occasion «fessue » et possède une grande poitrine donnant une forme disproportionnée. Par moment, c’est dans un foulard bien noué sur la tête que se cachent ces produits indésirables. Ces femmes vont plus loin en simulant une grossesse. A ce niveau, la trafiquante fait un beau paquet de produits prohibés qu'elle camoufle dans son jupon et se recouvre de pagnes qui donnent l'impression d'une femme qui porte une grossesse.

On a également la technique des emballages de jouets ou de biscuits. Ici, le trafiquant fait emballer les produits prohibés dans de grands paquets de biscuits ou de jouets hermétiquement fermés depuis le lieu d’achat. De 
cette façon, l'agent de contrôle se contentera de lire les écrits sur l'emballage. Avec les articles "fermés ${ }^{20}$, outre, les trous d'aérations et les réservoirs de carburants, sont utilisées des glacières neuves ou des valises neuves. Le trafiquant, après l'achat de ses articles, met à l'intérieur de la glacière ou de la valise des produits prohibés. Et une fois au bureau de douane, le dédouanement des articles visuels se fait selon les règles en vigueurs mais pas ceux qui sont à l’intérieur.

\section{Les transporteurs}

Ici, les acteurs sont à la fois transporteurs et contrebandiers. Ce dédoublement de rôles fait de ces derniers des pions efficaces en ce qui concerne le petit trafic de marchandises. En réalité, à une échelle réduite, les conducteurs servent souvent de relais pour les personnes qui ne peuvent pas se rendre à Osseïkro. Ils ramènent très souvent à celles-ci des produits de consommation courante comme le papier hygiénique, les savons, les boîtes d'allumettes, les téléphones portables, les matériels électroniques domestiques etc. D’autres transporteurs sont spécialisés dans le ravitaillement des vendeurs de carburant. A l'aide de leurs véhicules, dans la journée, ils partent plusieurs fois faire le plein de leur réservoir à Osseïkro. Puis une fois à Niablé, ils vident le contenu du réservoir dans un fût. Cette situation permet à la quasi-totalité des véhicules de la ville utilise le carburant d'origine ghanéenne.

Pour le transporteur, le montant du passage varie entre 2000 à 50 000f Cfa et il est fixé en fonction de la distance et de la nature de la marchandise. Pour rendre leur activité plus souple, les transporteurs ont une relation hypocoristique avec la quasi-totalité des agents de contrôle de Niablé et d’Abengourou. Par contre, les plus intrépides qui ont généralement un champ d'action plus vaste, n'hésitent pas à contourner les barrages de contrôle (voir la photo1) ou à utiliser plusieurs techniques de camouflages pour déjouer la vigilance des agents de douane. D’ailleurs, c'est avec la complicité de certains agents, nous avons déterminé quelques astuces de camouflages. En fait, ces transporteurs utilisent certains endroits de leur véhicule pour dissimuler les marchandises. Les endroits les plus fréquents sont le double fonds du vehicule, le siège du conducteur, les pneus de secours et chambre à air. En fait, dans le double fonds du véhicule, les objets non répréhensibles sont déposés de manière perceptible, les médicaments, la drogue sont camouflés au fond du véhicule dans un coffre spécialement aménagé, dans les poches des potières et souvent au niveau des moteurs.

Quant au siège du conducteur, très souvent exempts de contrôle, certains chauffeurs cachent sous leur siège spécialement aménagés, les

\footnotetext{
${ }^{20}$ Ici, il s’agit des émaillés, glacières, des valises, des sacs de voyage...
} 
produits prohibés. Concernant les pneus de secours et chambre à air, là, de faux pneus de secours sont vidés d'abord de leur chambre à air. On y introduit ensuite les produits prohibés tels que les médicaments, la drogue à l'intérieur puis on remet les chambres à air et on monte enfin les pneus.

\section{Les réseaux de contrebandiers}

Les différents réseaux contrebandiers ont un mode d’opération antinomique différent aux autres. Ils ont une organisation qui mobilise plusieurs acteurs intermédiaires qu'on trouve très souvent aux différents segments des chaînes de transition qui convergent vers Niablé. Leurs stratégies se déterminent en fonction des critères de coût et de minimisation des risques dans un environnement mouvant d'information imparfaite. Ces réseaux perdurent dans le temps lorsque les relations répétées durent entre les individus conscients de leur intérêt. D’ailleurs, pour atteindre leur objectif, certains préfèrent passer par les pistes contrebandières qui parsèment notre espace frontalier comme on le voit sur la photo 1. En réalité, ces pistes sont les principaux lieux de passages des marchandises contrebandières. Elles facilitent l'organisation et la mise en place de différents types de réseaux de contrebandiers dont deux sont constants dans notre espace frontalier.

Le premier type de réseau à un champ d’action peu réduit et moins puissant financièrement. C'est un groupe très hiérarchisé composé de courtiers, d'éclaireurs et de commerçants. Ici, les courtiers sont les pivots du trafic, ils organisent le trafic de sorte que la marchandise arrive à destination sans difficulté. Ils recrutent dans tous les milieux : commerçants, force de l'ordre, transporteurs, paysans, enseignants, etc. Ce qui leur permet de disposer de correspondants réguliers dans tous les secteurs. Ces correspondants leur donnent les renseignements concernant tous les mouvements endogènes et exogènes qui peuvent concourir au passage de leurs marchandises sans difficulté. Les éclaireurs, très souvent natifs de la localité sont des jeunes spécialisés dans la vérification des axes de contrôle et de l'indication des pistes clandestines. En effet, avant le passage des marchandises, les éclaireurs, sans marchandises, précédent les autres membres du groupe pour vérifier les points de contrôle. C'est après leur coup de fil que les autres membres décident de les suivre ou pas. Une fois passé, le rôle des courtiers et éclaireurs s’arrête. Les commerçants “prennent le relais en récupérant leurs colis dans les entrepôts affectés à cet effet.

Le deuxième réseau, le plus puissant financièrement, est également très structuré. Il est financé et réglementé par un homme d’affaire, appelé communément "transitaire" dans la ville. Pour minimiser les risques le transitaire divise son équipe en deux groupes. Le premier, convoyé par luimême, passe par la voie normale avec comme argument les pots-de-vin. 
Arrivé à chaque barrage, partant sur la base de la négociation, l’homme d'affaire finit par distribuer de l'argent aux agents de contrôle et/ou les autorités administratives et traditionnelles quand cela est nécessaire. Il fait ainsi passer ses marchandises sans aucun contrôle. Quant à son deuxième groupe, il achemine les marchandises sous la responsabilité d'un convoyeur. Avec la confiance du transitaire qui n'est pas du voyage, le convoyeur prépare l'arrivée des camions à la frontière. I1 entre en contact avec les douaniers ivoiriens qui sont au barrage de contrôle et les informe de la quantité et du type de marchandises qu'il désire faire passer. I1 convient avec eux d'une heure de passage et discute le prix de leur bienveillance qui se situe entre 100000 et $1000000 \mathrm{f}$ cfa selon le tonnage et le nombre des véhicules. Les négociations terminées, le convoyeur avertit les chauffeurs qui quittent les entrepôts avec des camions bien chargés. (Voir la photo2).

Ces diverses techniques de camouflage utilisées par les contrebandiers nous ont freinés dans notre quête d'évaluation des volumes de charges en transit. De la même façon, il paraît compliqué d'évaluer les dividendes de la contrebande et de les comparer avec celles dérivées d'autres activités frontalières.

$\mathrm{Au}$ total, force est de reconnaître que la fertilité imaginative caractérise les contrebandiers car nul ne peut prétendre cerner les astuces qu'ils utilisent. Au regard, de toutes ces astuces, seuls l'intuition, le hasard et quelques fois l'odeur des produits prohibés guident les forces de l'ordre dans leur contrôle. Ce qui leur permet de saisir des fois d'importantes marchandises comme le montre le tableau ci-dessous.

Tableau 2: Marchandises saisies par la Douane de Niablé.

\begin{tabular}{|ll|}
\hline Marchandises saisies & Quantité \\
\hline Friperie & $\mathbf{5 2 1}$ balles \\
\hline Tronçonneuse & $\mathbf{2 1}$ unités \\
\hline Sucre & $\mathbf{8 4}$ sacs \\
\hline Cacao & $\mathbf{4 5 2}$ sacs \\
\hline Produits phytosanitaires & $\mathbf{9 4}$ cartons \\
\hline Boissons alcoolisées & $\mathbf{3 4 6}$ cartons \\
\hline \hline Produits pharmaceutiques & $\mathbf{7}$ cartons \\
\hline
\end{tabular}

Source : Bureau des Douanes de Niablé, Aout 2012.

A l'observation de ce tableau, les saisies sont dominées respectivement par la friperie, les sacs de cacao et les boisons alcoolisées. Tous ces produits sont difficilement camouflables puis leur passage se fait en quantité importante. Donc une saisie constitue un butin élevé pour la douane nous fait remarquer un agent de douane. 


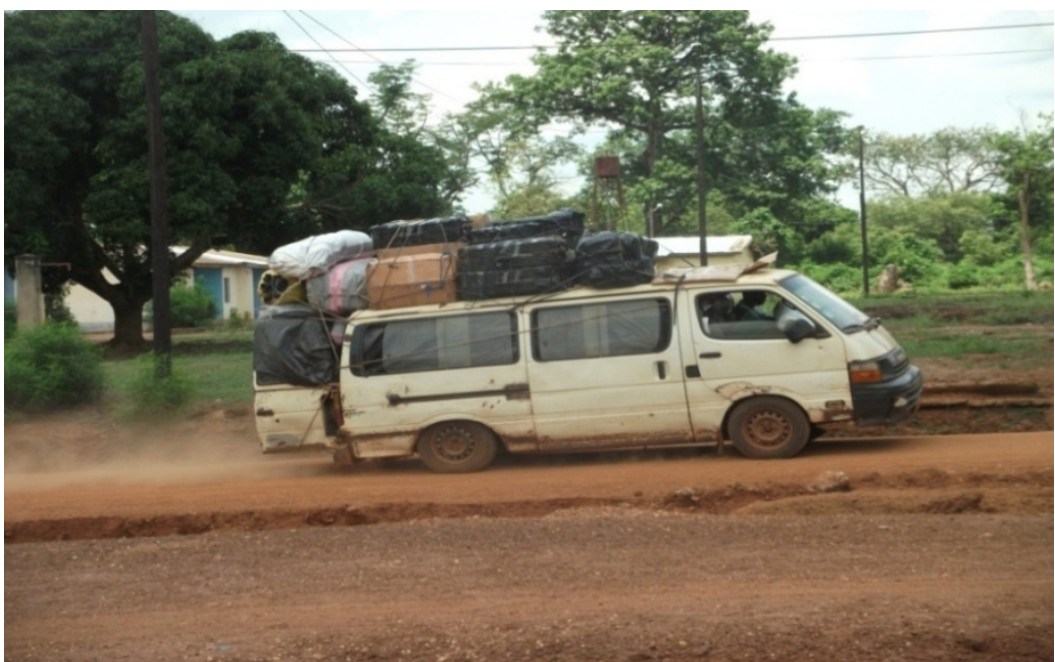

Photo 1 : Un véhicule provenant du Ghana tente d'échapper aux barrages de contrôle en passant par l'une des pistes clandestines de l'axe Osseïkro-Zouhounou

(Cliché KOUADIO Datté .A, Aout 2012)

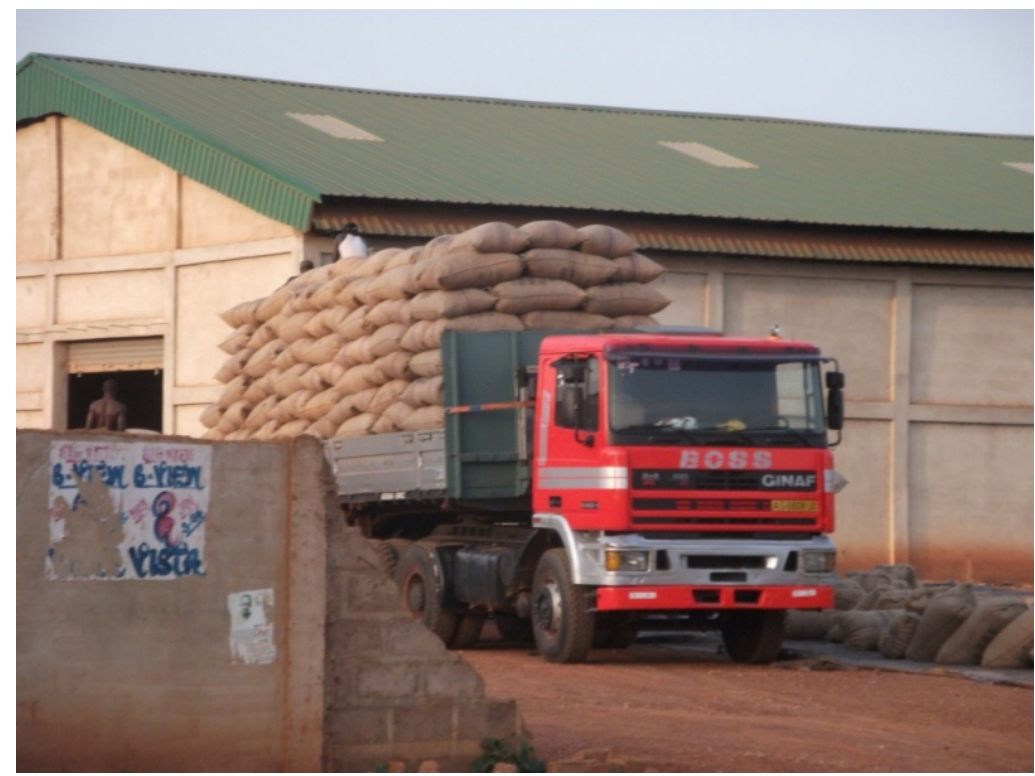

Photo2: Un véhicule appartenant à un transitaire charge les sacs de cacao dans un entrepôt à Niablé. Même chargé, le chauffeur attendra le coup de fil du convoyeur pour quitter la ville.

(Cliché KOUADIO Datté .A, Aout 2012)

Les effets de la contrebande sur la ville de Niablé Les effets socio-économiques de la contrebande

A Niablé, la contrebande contribue incontestablement à résorber relativement le chômage. Car cette activité permet à ces affiliés de survivre et de s'insérer dans le tissu social. Elle modifie ainsi leur vie et celle de toute 
la population frontalière vu qu'elle lui fournit des ressources indispensables pour sa survie. Dans la localité, on a l'impression que cette activité illicite se déroule à sens unique car la plupart les marchandises ont pour destination finale le territoire ivoirien. D’ailleurs, les articles qui dominent cette activité sont dans les domaines de la quincaillerie, les produits cosmétiques, la bonneterie, les boissons alcoolisées et tabacs, les produits d'équipements électroménagers, les produits alimentaires, les articles électriques et électroniques, la friperie, les produits pétroliers et pharmaceutiques. Cependant la consommation de ces produits contrebandiers, notamment alimentaires et pharmaceutiques menace la santé de la population zone frontalière. En fait, la consommation des produits alimentaires peut donner lieu à de graves problèmes d'intoxication, en particulier les produits périmés et qui peuvent nuire à la santé des consommateurs. Concernant les médicaments importés, leur consommation constitue également un danger sur la santé des habitants vu l'accès libre et illimité à toutes les classes médicamenteuses avec une méconnaissance totale de la part du vendeur et de l'acheteur. Pis encore, les conditions de transport, de stockage et de vente des médicaments sont les mêmes que toutes celles des autres marchandises de contrebande. Aucun traitement en faveur ne leur est accordé. Ils sont transportés avec les bidons de carburant, cachés à l'intérieur des sacs de farine, stockés avec les dattes et les grandes marmites d'aluminium et vendus avec des insecticides.

Par ailleurs, la commercialisation de ces marchandises issue de la contrebande freine le développement économique normal de Niablé en renforçant la désarticulation de l'économie urbaine. En fait, la contrebande est devenue une véritable «industrie» qui a mis à plat toutes les formes légales de commerce de la zone. Outre, des produits habituels, c'est le déferlement surtout de produits pétroliers, médicamenteux et des produits chinois qui arrivent en force, lesquels annoncent le coup de massue pour certaines variétés de produits nationaux. En effet, la contrebande favorise aussi le développement d'un marché de change parallèle. A Niablé, si le commerce informel reste très florissant pour les contrebandiers, le ralentissement général de l'activité pèse lourdement sur les acteurs du secteur formel.

\section{L' impact spatial de la contrebande sur la ville de Niablé.}

L'impact spatial se traduit par l'extension de la surface urbanisée par les acteurs directs ou indirects de la contrebande. A Niablé, les contrebandiers natifs de la ville éprouvent une nécessité d'investir dans l'immobilier. En fait, eu égard à leur activité, ils bénéficient de meilleurs matériaux de construction et à moindre coût. Les plus nantis ont des maisons types villa moderne dans les nouveaux quartiers de la ville. Il existe même 
un véritable challenge entre eux pour la construction de la plus belle villa. Selon eux, la qualité et la quantité de villas réalisées démontrent la bonne santé de leur pouvoir économique. Mais tout ceci est de bonne guerre car cela améliore l'image urbaine. Quant au moins nantis, attentifs aux opportunités économiques et notamment foncières, ils accèdent l'achat de terrains ou à la rénovation des veilles habitations et à l'installation de nouvelles boutiques et magasins.

Toutefois, la contrebande porte atteinte au développement de Niablé. Au niveau environnemental, en dépit d’absence d’industrie à Niablé, les risques naturels de pollution sont importants. Cela s'explique par la forte présence des produits pétroliers non raffinés qui polluent considérablement l'air. En plus, ces produits présentent d'énormes risques d'accidents tels que les incendies. Même si pour le moment, aucun cas d'incendie n’a été signalé dans ce sens, ce serait la catastrophe si cela devait se produire car le liquide inflammable se vend près des habitations. Faut-il le rappeler, Niablé ne possède pas de station service. Toute tentative d'implantation de stations services est vouée à l’échec vu la préférence du carburant d’origine ghanéenne par les consommateurs.

\section{Conclusion}

La contrebande est un sujet complexe et sensible dont de nombreux aspects et ceux qui y sont impliqués sont tenus secrets. Alors, à Niablé, l'évoquer c'est prendre en compte les facteurs socioculturels et économiques qui aident les contrebandiers à établir une typologie de réseaux bien organisés. Ceux-ci adoptent plusieurs stratégies pour donner plus de vitalité à cette activité illicite afin de s’insérer dans le tissu socio-économique. Outre, une telle organisation permet aux contrebandiers de dégager des marges importantes qu'ils répercutent sur la mutation socio-spatiale de la ville.

Toutefois, cette activité illicite fait apparaître des effets parasitaires dans la localité qui ne sauraient constituer une alternative au développement de Niablé. C’est pourquoi, nous suggérons aux autorités compétentes de mener une lutte hardie contre cette activité illicite qui risque d'hypothéquer non seulement l'avenir de la ville de Niablé mais aussi de l’économie nationale.

\section{References:}

AHOUSSI J., 2008, Le commerce transfrontalier et le développement urbain : cas de Noé. Mémoire de maîtrise, Université de Cocody-Abidjan, IGT, 104 p.

AKA K., 2008, Les taxi-motos dans une ville frontalière de Côte d'Ivoire: Niablé. Université de Cocody-Abidjan, CRAU, 22 p.

ATTA K, 1989, Echanges régionaux, commerce frontalier et sécurité 
alimentaire en Afrique de l'Ouest: Rapport d'enquête sur les marchés frontaliers ; les échanges Côte d'Ivoire-Mali, Paris, OCDE, club du sahel, ORSTOM -Abidjan, 45 p.

N BENNAFLA K., 2002, Le commerce frontalier en Afrique centrale. Acteurs, espaces, pratiques. Paris, Karthala, 368 p.

GREGOIRE E., 1996, Les chemins de la contrebande. Etude de réseaux commerciaux en pays houssa, Cahiers d'études africaines, $n^{\circ} 124$, pp. 509532.

IGUE J O., 1977, Le commerce de contrebande et les problèmes monétaires en Afrique de l'Ouest , CEPFAP, Cotonou (Bénin, Université Nationale du Bénin).

KOFFI H, 2004, Perméabilité des frontières ivoiriennes : le cas de Niablé. Mémoire de Maîtrise, Université d'Abidjan Cocody, UFR Criminologie, 79p. KOUADIO D, 2006, Niablé, une ville frontalière : impact d'une économie basée sur les échanges transfrontaliers et sur le dynamisme de la ville, Mémoire de Maîtrise de Géographie, IGT/Université de Cocody, Abidjan, 116 pages.

NASSA D., 2005, Commerce transfrontalier et structuration de l'espace au nord de la Côte-d'Ivoire. Thèse de doctorat, Université de Bordeaux 3, 336 p.

STARY .B., 1994, Une petite ville ivoirienne dans sa spécificité géographique : Niablé, étude d'un espace frontalier, Notes et travaux (Groupement Interdisciplinaire en Sciences Sociales de Côte-d'Ivoire) $n^{\circ} 4$, Abidjan : Orstom, 41 p. 